\title{
Reliability Assessment of Distribution Systems Incorporating Feeder Restoration Actions
}

\author{
Fabio D’Agostino, Federico Silvestro \\ Department of Electrical, Electronic, Telecommunication \\ Engineering and Naval Architecture \\ University of Genova \\ Genova, Italy \\ fabio.dagostino@edu.unige.it, federico.silvestro@unige.it
}

\author{
Kevin P. Schneider \\ Battelle Seattle Research Center \\ Pacific Northwest National Laboratory \\ Seattle, WA, USA \\ kevin.schneider@pnnl.gov
}

\begin{abstract}
This paper proposes a computational methodology for the evaluation of the IEEE reliability indices for distribution systems considering distribution system restoration. The goal of the proposed methodology is to move from a reliability assessment based on historical data to a computational approach. The developed tool allows the evaluation of the Service Restoration benefits, in terms of customers interruption duration in case of fault occurrences. Distribution System Restoration (DSR) is aimed at restoring loads after a fault by altering the topological structure of the distribution network while meeting electrical and operational constraints. The Spanning Tree Search algorithm is used to identify a post-outage topology that will restore the maximal amount of load with a minimal number of switching operations. The goal of the proposed tool is to determine the optimal switching sequences for the restoration process. The reliability indices incorporates contributions of all possible faults effects.
\end{abstract}

Index Terms-- Distribution System Reliability, Distribution System Restoration, Space State Tree.

\section{INTRODUCTION}

Reliability enhancement is an important goal for the development of smart distribution systems [1]. Distribution system reliability can be improved by installing Distribution Automation (DA) infrastructures, such as Advanced Metering Infrastructure (AMI) and Remote Controlled switches (RCSs), which enable remote monitoring and control capability [2]. Smart grid algorithms also help in the enhancement of reliability. For example, fault location, isolation, and service restoration (FLISR) enables fast detection of faults and enhances the ability to quickly recover from disruptions [3].

This work was supported by Office of Electricity Delivery and Energy Reliability, U.S. Department of Energy (DOE) and Pacific Northwest National Laboratory (PNNL).

\author{
Chen-Ching Liu, Yin $\mathrm{Xu}$ \\ School of Electrical Engineering and \\ Computer Science \\ Washington State University \\ Pullman, WA, USA \\ liu@eecs.wsu.edu,yxu2@eecs.wsu.edu
}

\author{
Dan T. Ton \\ Office of Electricity Delivery and Energy Reliability \\ U.S. Department of Energy \\ Washington, DC, USA \\ dan.ton@hq.doe.gov
}

In practice, the balance between reliability and cost is important. It is not economically justifiable to pursuit high reliability without considering the costs. Therefore, a comprehensive cost-benefit analysis will need to be performed before a new technology is put into use. In order to evaluate the benefit for reliability gained from a smart grid technique, reliability indices will be calculated for the target distribution system with and without the new technology, respectively, and then the results are compared. A computational tool that is able to assess the reliability of a distribution system with smart grid techniques, such as distribution service restoration (DSR), is necessary to perform the benefit analysis.

Reliability evaluation methods [4] can be categorized into analytical methods, e.g., failure mode and effect analysis (FMEA) [5], and time-sequential simulation techniques, e.g., Monte Carlo simulation [6]. The existing distribution system analysis tools adopted by industry, e.g., Synergi Electric Software [7], evaluate the reliability based on a fix radial topology. It is assumed that, a fault on a component will cause interruption to all customers downstream the component and the customers will not be restored until the faulted component is repaired. The effect of DSR is not taken into account.

Some recent studies have considered the effect of a special kind of DSR strategies on distribution system reliability [8][10]. In these studies, the DSR strategy is simply closing one normally open tie switch to restore all or a portion of customers in an interrupted area. The control method of switches, i.e., manual or remote-controlled, is also considered. In our prior work [11], a reliability analysis method is proposed to calculate the System Average Interruption Frequency Index (SAIFI) and System Average Interruption Duration Index (SAIDI) for distribution systems with DSR. The method is applied to the evaluation of RCS placement schemes in [12].

This paper proposes a novel approach to distribution system reliability assessment, which considers DSR and remote control capability. The new tool is based on FMEA. The universal set 
of single fault events are considered. It takes the outputs of the DSR algorithm, i.e., the switching operations for service restoration, as inputs. For each event, the optimal switching sequence is determined based on the state transition graph and the outage duration for each customer is calculated according to the switching sequence and the switching time of each switch. The contribution of each single fault event to the reliability indices is calculated separately. Joining with the failure rate, reliability indices for the entire system are determined.

The remainder of this paper is organized as follows. Section II describes the Reliability Assessment Tool, introducing the algorithm the Reliability Indices and the Distribution System Restoration tool. Section III reports the proposed methodology for the Reliability Analysis problem. Section IV illustrates the solution method. Sections V and VI are dedicated to the testing results and conclusions, respectively.

\section{RELIABILITY ASSESMENT TOOL}

\section{A. Tool Design}

The Reliability Analysis (RA) method takes the information of the target distribution system and the output of the Distribution System Restoration (DSR) tool as inputs. A target reliability index needs to be set for the RA method. The outputs of the RA method include the optimal switching sequence for the set of fault scenarios of interest and the values of several common used reliability indices, computed over a specified period of time. Figure 1 shows the block diagram of the proposed method.

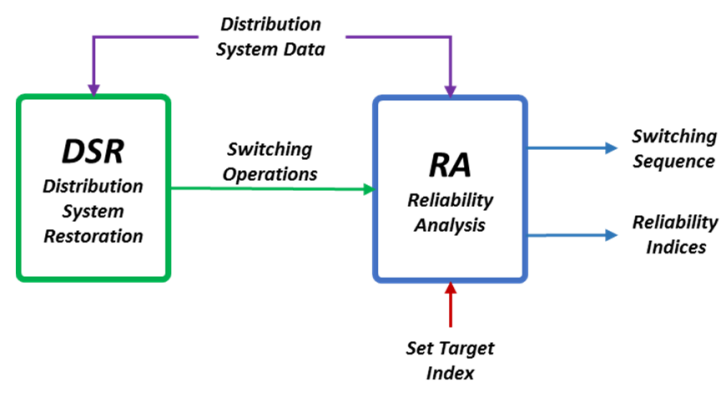

Figure 1 - Design of the reliability analysis tool.

Here, the distribution system information includes the topology, load at sections, locations and status of switches, generation capacity of Microgrids, etc. The DSR tool provides the final restoration scheme for fault scenarios of interest. The switching sequence will be determined by the RA method, which will minimize the selected target index.

\section{B. Reliability Indices}

The IEEE Guide for Electric Power Distribution Reliability Indices [13] identifies distribution reliability indices and factors that affect their calculation. Six standard indices are selected for the reliability assessment:

- $\quad$ SAIFI - System Average Interruption Frequency Index

- $\quad S A I D I-$ System Average Interruption Duration Index

- $\quad$ CAIDI - Customer Average Interruption Duration Index

- $A S A I-$ Average Service Availability Index
- $\quad A S I F I-$ Average System Interruption Frequency Index

- $A S I D I$ - Average System Interruption Duration Index

SAIDI and ASIDI can be selected as target indices. The first index is the average duration of interruptions for all customers during a predefined period of time.

$$
S A I D I=\frac{\sum r_{i} N_{i}}{N_{T}}
$$

- $r_{i}$ minutes of interruption

- $N_{i}$ total number of customers interrupted

- $\quad N_{T}$ total number of customers served

The second one is based on load rather than customers affected.

$$
A S I D I=\frac{\sum r_{i} L_{i}}{L_{T}}
$$

- $r_{i}$ minutes of interruption

- $L_{i}$ total nterrupted load

- $\quad L_{T}$ total served load

These two indices are commonly measured in minutes or hours of interruption.

\section{Distribution System Restoration}

Distribution System Restoration (DSR) is aimed at restoring loads after a fault by altering the topological structure of the distribution network while meeting electrical and operational constraints. With DSR, all or a portion of interrupted load can be restored through neighboring feeders by reconfiguration, taking into account the possible presence of Microgrids. As a result, the outage duration is reduced. The reliability is improved [11].

A spanning tree search algorithm is proposed to determine the optimal restoration strategy [14]. Distribution systems are represented by spanning trees. A spanning tree is a graphtheoretic term that refers to a graph without loops that covers all nodes in the system. A search procedure is designed to identify the post-outage distribution system topology that will restore the maximal amount of load with a minimal number of switching operations. The spanning tree search algorithm is used in this work to generate DSR strategies.

\section{PRoposed Methodology}

The DSR tool defines the switches to operate, identifying the final restoration schemes of the network, for all possible faults. However, the switching sequences are unknown and the restoration process has to be made following only operational rules by system operators.

The purpose of the proposed methodology is to compute the optimal switching sequence while introducing the selected reliability index as the objective function. Results of the tool are the optimal switching sequence and the reliability indices, by implementing the DSR actions.

For the purpose of the paper, the term event indicates the loss of a load zone as a consequence of a fault.

\section{Problem definition}

The proposed methodology is based on the definition of restoration process as a sequence of states described by the status of the switches. Considering $n$ switches identified by the 
DSR tool, it is possible to define a state space of $n^{2}$ dimension. This approach allows the formulation of the problem using graph techniques.

The following objects have been defined:

1) State - $K_{k} \mid S_{1}, S_{2}, \ldots, S_{n}$

Each state identifies the grid in the configuration defined by the switches status. Every state is characterized by:

- Status of the switches to operate $-S_{1}, S_{2}, \ldots, S_{n}$

- Total interrupted load - $L_{k}$

- Total number of interrupted customers - $N_{k}$

It is important to notice that the considered switches are the ones identified by the restoration tool; all other switches are considered to have their status fixed during the restoration process.

\section{2) Transition Arc - $t_{p, c}$}

Represents the time needed for the transition between two states, from the parent state $(p)$, to the child state $(c)$. The time is defined by the Mean Time to Operate (MTTO) of the switches and it is related to the kind of switch (manual or remote), the coordination time with the control center, the location of substations and the time required for the physical operation.

\section{3) Level - $l$}

The level identifies the number of operations during the restoration process and represents the number of single switching operation required for obtaining the considered state starting from the initial state. The initial state is defined by the status of the switches in the post-fault condition.

Figure 2 shows the states $K_{5}$ and $K_{1}$, connected by a 40 minutes transition arc. Each state is defined by the total amount of disconnected load $(380 \mathrm{~kW}$ and $500 \mathrm{~kW}$, respectively) and the numbers of unsupplied customers (100 and 400). The transition between two states is available through the operation of the $S_{2}$ switch, that allow transition from level 2 to level 3.

In order to simplify the graph representation, every state $K_{k}$ is identified by the decimal conversion of the switch status, as a binary number (e.g. $\left.K_{2} \mid \begin{array}{llll}0 & 0 & 1 & 0\end{array}\right)$.

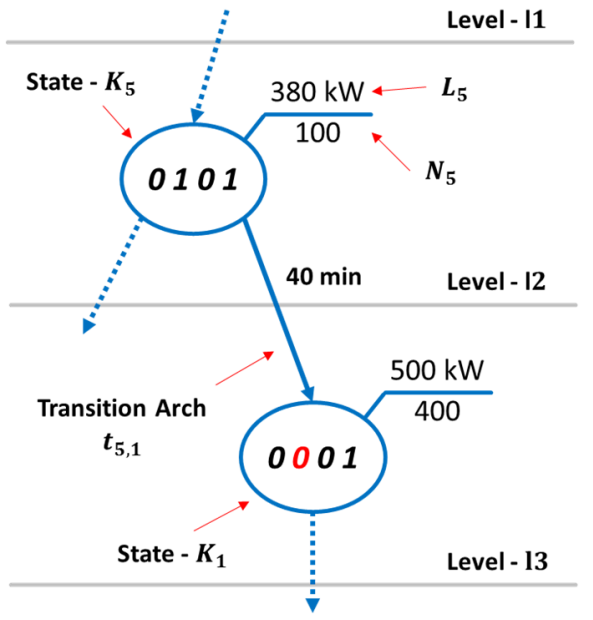

Figure 2 - Graph representation

\section{E. Graph generation}

The problem formulation is subject to electrical and operational constraints. The graph generation is composed of two steps:

- State Space generation

- Arcs generation

The first step defines the parameters for each state, evaluating the grid topology for each state. The second step considers connection arcs between the defined states.

The arcs generation is subject to the following rules and operational constraints:

- Using the switches status as Boolean variables, the final state can be written as shown in equation (3). The DSR tool identifies the switches that have to be operated. Therefore, once the initial state is defined, the final state is automatically defined. That is,

$$
K_{f}=\bar{K}_{\iota}
$$

- Simultaneous switching operations are not allowed: arcs connect states that only belong to sequential levels.

- Multiple operations on the same switch are not allowed: the total number of operation is equal to the number of switches.

$$
n_{o p}=n
$$

- A sequence of switching operations is a possible solution if all the states in which the system transits are feasible. Connections between states exist only if the states are both feasible. Several conditions are added for the feasibility check. In this research, it is considered that the total supplied load is less than the total generation capacity. Equations (5) and (6) show the conditions set of Feeder $F$ and the set of Microgrids $M$.

$$
\begin{array}{cc}
P_{k}^{f} \leq P_{k}^{f \max } & \forall f \in F \\
P_{k}^{m} \leq P_{k}^{m \max } & \forall m \in M
\end{array}
$$

- The supplied zones remain active during the restoration process. For each state of the solution path, the set of unsupplied zone has to be a subset of the initial one.

- The zone affected by the fault has to remain unsupplied during the restoration process. Arcs cannot connect states in which the fault zone appears supplied.

- The feeders have maintain the radial configuration during the restoration process. The only exception allowed is the load transfer between two different sources. After the load is pick up, the mesh structure has to be removed: arcs cannot connect two sequential meshed states.

\section{$F$. Objective function - Target Index}

The solution process has to solve the hybrid graph minimizing the objective function subject to the defined constraints. According to IEEE indices previously presented, it is possible to define several objective functions, according to the main target of the restoration process.

Starting from the definition of index and considering the contribution of the single event, it is possible to define the 
event as a sequence of different states of the grid, characterized by the switch status and unsupplied load and customers.

Defining $p$ as the solution path that represents the sequence of $n$ states, it is possible to write equations (7) and (8), as the objective functions for the two target indices: SAIDI and ASIDI respectively.

$$
\begin{aligned}
& F^{S A I D I}=\sum_{j=1}^{n} \frac{r_{\boldsymbol{k}} N_{\boldsymbol{k}}}{N_{T}} \\
& F^{A S I D I}=\sum_{j=1}^{n} \frac{r_{\boldsymbol{k}} L_{\boldsymbol{k}}}{L_{T}}
\end{aligned}
$$

Where:

$$
\begin{array}{ll}
- & k=p(j) \\
- & r_{\boldsymbol{k}}=t_{\boldsymbol{p}(\boldsymbol{j}) \boldsymbol{p}(\boldsymbol{j}+\mathbf{1})}
\end{array}
$$

The state $k$ is the state in which the system transits during the restoration process, at step $j$. The transition time $r_{\boldsymbol{k}}$ is defined by the weights of the arc that connects the consequential states.

\section{G. Event contribution}

After the restoration process, a fault has to be removed and it is necessary to consider the Mean Time to Repair (MTTR), related to the zone affected by the fault.

Due to the fault location, it is possible to have full or partial restoration. The last state of the restoration process shows the residual number of customers disconnected $N_{p(n)}$ and the total unsupplied load $L_{p(n)}$. These contributions have to be considered in the evaluation of indices.

Equations (9) and (10) show respectively the expressions of the contributions to the SAIDI and the ASIDI indices, considering the fault of zone $i$.

$$
\begin{aligned}
& S A I D I_{Z i}=F_{Z i}^{S A I D I}+\frac{\operatorname{MTTR}_{Z i} \cdot N_{p(n)}}{N_{T}} \\
& A S I D I_{Z i}=F_{Z i}^{A S I D I}+\frac{M T T R_{Z i} \cdot L_{p(n)}}{L_{T}}
\end{aligned}
$$

\section{H. Reliability evaluation}

By the proposed methodology it is possible to study the effect of every single fault (loss of each zone), evaluating the index contributions of the zone loss, considering the DSR actions. The computation of the original indices provides a "measure" of the DSR benefits, showing the decreasing of them due to restoration strategies.

After the computation of every fault contribution, it is possible to define the original indices considering the number of occurrences of each fault over a defined period.

The number of fault occurrences over one year of service, for each zone, is the failure rate $(\lambda)$ of the zone. Expressions (11) (12) report the SAIDI and ASIDI indices, considering one year of service.

$$
S A I D I=\sum_{i=1}^{n_{Z}} \lambda_{Z i} \cdot S A I D I_{Z i}
$$

$$
A S I D I=\sum_{i=1}^{n_{Z}} \lambda_{Z i} \cdot A S I D I_{Z i}
$$

Where:

$$
\begin{array}{lll}
\text { - } & n_{Z} & \text { number of zones } \\
\text { - } & \lambda_{Z i} & \text { failure rate of zone } i \\
\text { - } & S_{A I D I_{Z i}} & \text { contribution of zone } i \\
\text { - } & A_{S I D I_{Z i}} & \text { contribution of zone } i
\end{array}
$$

\section{SOLUTION METHOD}

The grid model and the DSR output represent the main input for the RA method. The failure rates, the MTTR of each zone and the MTTO of the switches complete the input data set, adding the information required for the reliability assessment.

The DSR output is organized in two stages; Every stage identifies a different graphs, which is computed following the rules defined in section III:

- Stage 1: operates only RCS to restore as much load as possible. The main purpose of the first stage is to close the feeder breaker after tripping due to the fault, by using remote control technology for isolating the zone affected by fault.

- Stage 2: uses all available switches to restore the rest of interrupted load. The second stage is slower than the first one, due to the manual operation times.

The Shortest Path Algorithm [15] has been used for the solution procedure. Dijkstra's method [16] has been adopted, assuming the weights of the edges to be positive values in a sparse matrix, which represents the graph.

Therefore each hybrid graph needs to be converted in a sparse matrix, in which the non-zero entries are related to the arcs of the original graph. The states represent the vertices and the edge are computed according to the selected target index, through the multiplication of the state parameters with the transition time of the arcs.

If the target is the SAIDI index, the weight of the edges is computed by multiplying the number of unsupplied customer with the time associated to the transition arcs. If the target is the ASIDI index, the weight of each edge is calculated using the total unsupplied load of the parent state.

For clarity, the solution process for the zone 89 fault is here described in details, in the following.

The simulation grid is the 4-feeder 1069-node unbalanced test system developed by Pacific Northwest National Laboratory (PNNL) [17].

Figure 5 shows the topology of the test grid modeled by a graph. Vertices represent the switches and the edges represent the load zones. Red circles indicate the RCSs available: four automatic feeder breakers, four Microgrid interfaces and two tie-breakers. Switches are identified by the two connected zones; green and blue markers indicate the stage one and stage two switches respectively. Numbers indicate the operation sequence, defined by the RA method.

This section reports the detailed solution process for the failure of zone 89. In the post fault condition the Feeder 
Breaker c (FB-c) is considered open, as a consequence of the protection intervention, and Microgrid 3 is considered islanded, as consequence of the self-protection system action.

Table I shows the DSR output for the selected zone, i.e. the list of switches to operate. As previously introduced, stage 1 considers only RCS to restore as much load as possible and stage 2 identifies all available switches to restore the rest of interrupted load.

The MTTO of switches is 30 minutes for manual switches and 20 seconds for RC switches.

TABLE I - DSR OUTPUT

\begin{tabular}{|c|c|}
\hline Stage 1 & Stage 2 \\
\hline$[96$ 89] & {$[4647]$} \\
{$[\mathrm{Fc} 81]$} & {$[5396]$} \\
{$[90106]$} & {$[8990]$} \\
{$[\mathrm{M} 393]$} & {$[4590]$} \\
\hline
\end{tabular}

Figure 3 shows the Stage 1 graph, defined by the four RC switches. The state space has dimension $n$ equal to 16 . The number of disconnected customers and the total unsupplied load characterize each state.

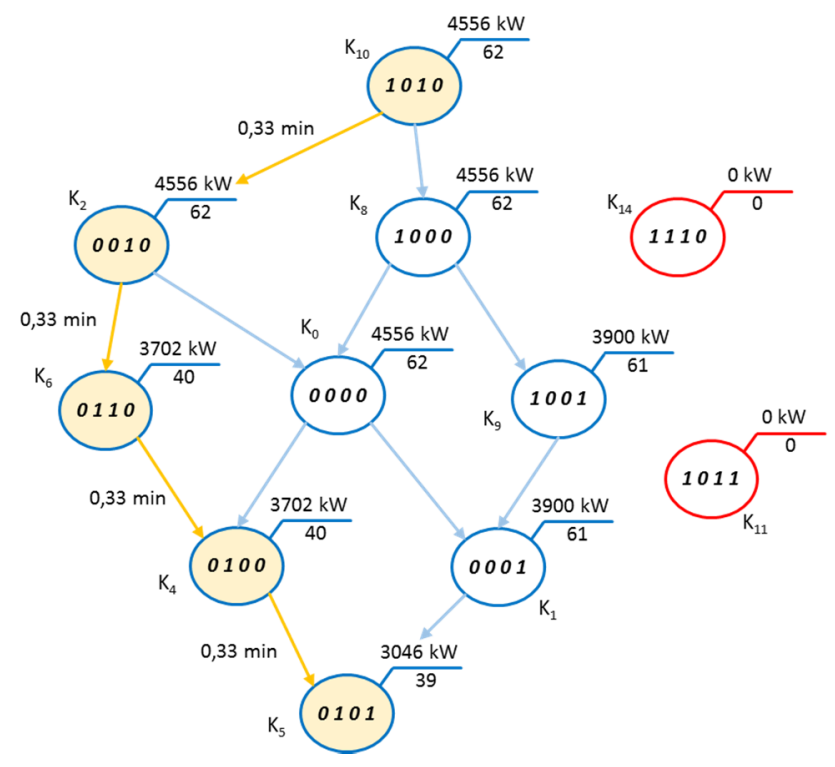

Figure 3 - Stage 1 graph

Connections between states exist only if the operational constraints are satisfied and only between states belonging to sequential level. Figure 3 depicts the graph of the stage 1 problem. Red circles show a part of the infeasible states.

The SAIDI index is selected as a target and the yellow path represents the solution of the graph obtained through the Shortest Path algorithm. Equation (13) reports the solution path of the graph, i.e., the sequence of states that minimizes the target index.

$$
p_{1}=\{10,2,6,4,5\}
$$

Table II reports the parameters of the states used in the graph generation. Feasibility and meshing columns have entries equal to 1 when the condition is verified.
TABLE II - StAGE 1

\begin{tabular}{|c|cccc|c|c|c|c|}
\hline $\mathbf{k}$ & $\mathbf{S}_{\mathbf{1}}$ & $\mathbf{S}_{\mathbf{2}}$ & $\mathbf{S}_{\mathbf{3}}$ & $\mathbf{S}_{\mathbf{4}}$ & $\mathbf{N}_{\mathbf{k}}$ & $\mathbf{L}_{\mathbf{k}}[\mathbf{k W}]$ & Feasibility & Meshing \\
\hline 0 & 0 & 0 & 0 & 0 & 62 & 4556 & 1 & 0 \\
1 & 0 & 0 & 0 & 1 & 61 & 3900 & 1 & 0 \\
2 & 0 & 0 & 1 & 0 & 62 & 4556 & 1 & 0 \\
3 & 0 & 0 & 1 & 1 & 22 & 854 & 0 & 0 \\
4 & 0 & 1 & 0 & 0 & 40 & 3702 & 1 & 0 \\
5 & 0 & 1 & 0 & 1 & 39 & 3046 & 1 & 0 \\
6 & 0 & 1 & 1 & 0 & 40 & 3702 & 1 & 0 \\
7 & 0 & 1 & 1 & 1 & 0 & 0 & 0 & 0 \\
8 & 1 & 0 & 0 & 0 & 62 & 4556 & 1 & 0 \\
9 & 1 & 0 & 0 & 1 & 61 & 3900 & 1 & 0 \\
10 & 1 & 0 & 1 & 0 & 62 & 4556 & 1 & 0 \\
11 & 1 & 0 & 1 & 1 & 0 & 0 & 0 & 0 \\
12 & 1 & 1 & 0 & 0 & 1 & 656 & 0 & 0 \\
13 & 1 & 1 & 0 & 1 & 0 & 0 & 0 & 0 \\
14 & 1 & 1 & 1 & 0 & 0 & 0 & 0 & 0 \\
15 & 1 & 1 & 1 & 1 & 0 & 0 & 0 & 0 \\
\hline
\end{tabular}

Equation (14) reports the total weight of the solution path, i.e. the first contribution of the considered fault to the SAIDI index.

$$
S A I D I_{Z 89-S T A G E 1}=0.274 \mathrm{~min}
$$

The switching sequence $p_{1 s}$ is automatically defined by the solution path:

$$
p_{1 s}=\{[96 \text { 89], [Fc 81], [90 106], [M3 93] }\}
$$

The first operation is to open switch [96 89] that allows restoring the portion of the feeder upstream the fault, closing feeder breaker [ $\mathrm{Fc} 81]$. The third switching operation is to open switch [90 106], which allows supplying of zones 93,106 and 107 by closing the microgrid switch [M3 93].

The second stage of the DSR output identifies the graph proposed in Figure 4.

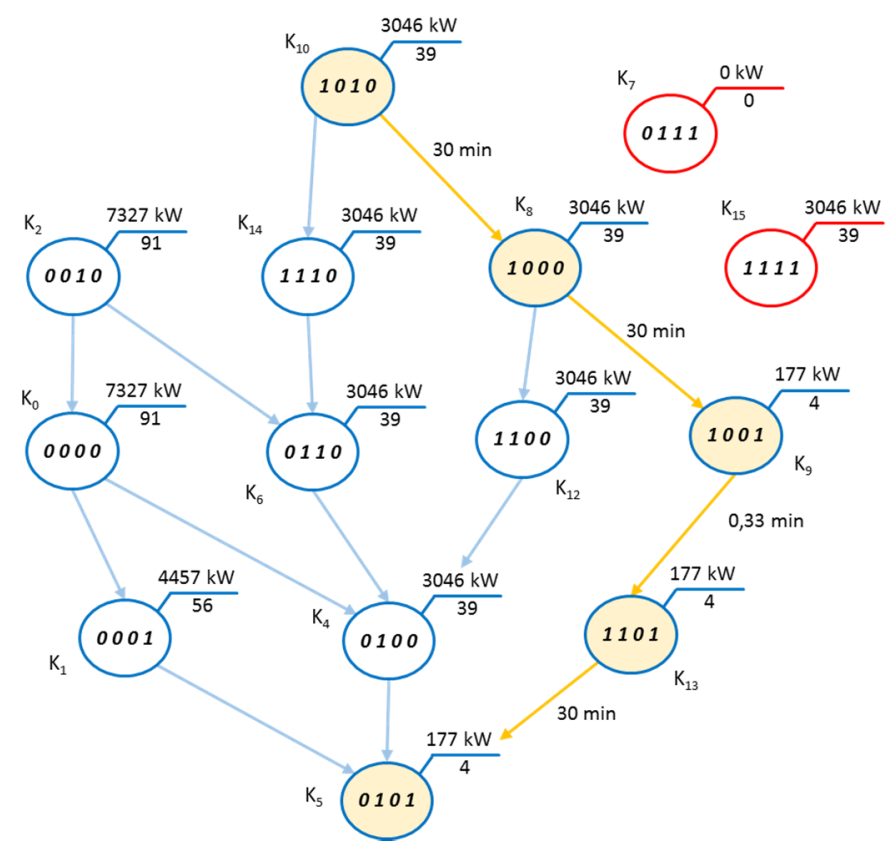

Figure 4 - Stage 2 graph

Equation (16) reports the solution path of the second stage, which represents again the optimal switching sequence, defined by the transition between the highlighted states. 


$$
p_{2}=\{10,8,9,13,5\}
$$

Table III reports the parameters of states used in the graph generation. It is important to note that the parameters of the last state of stage 1 are the same as those of the first state of the stage 2. Although the two states appear to be different as they are defined by different switches, the two states are in fact the same.

TABLE III - STAGE 2

\begin{tabular}{|c|cccc|c|c|c|c|}
\hline $\mathbf{k}$ & $\mathbf{S}_{\mathbf{1}}$ & $\mathbf{S}_{\mathbf{2}}$ & $\mathbf{S}_{\mathbf{3}}$ & $\mathbf{S}_{\mathbf{4}}$ & $\mathbf{N}_{\mathbf{k}}$ & $\mathbf{L}_{\mathbf{k}}[\mathbf{k W}]$ & Feasibility & Meshing \\
\hline 0 & 0 & 0 & 0 & 0 & 91 & 7327 & 1 & 0 \\
1 & 0 & 0 & 0 & 1 & 56 & 4457 & 1 & 0 \\
2 & 0 & 0 & 1 & 0 & 91 & 7327 & 1 & 0 \\
3 & 0 & 0 & 1 & 1 & 52 & 4280 & 0 & 0 \\
4 & 0 & 1 & 0 & 0 & 39 & 3046 & 1 & 0 \\
5 & 0 & 1 & 0 & 1 & 4 & 177 & 1 & 0 \\
6 & 0 & 1 & 1 & 0 & 39 & 3046 & 1 & 0 \\
7 & 0 & 1 & 1 & 1 & 0 & 0 & 0 & 0 \\
8 & 1 & 0 & 0 & 0 & 39 & 3046 & 1 & 0 \\
9 & 1 & 0 & 0 & 1 & 4 & 177 & 1 & 0 \\
10 & 1 & 0 & 1 & 0 & 39 & 3046 & 1 & 0 \\
11 & 1 & 0 & 1 & 1 & 0 & 0 & 0 & 0 \\
12 & 1 & 1 & 0 & 0 & 39 & 3046 & 1 & 1 \\
13 & 1 & 1 & 0 & 1 & 4 & 177 & 1 & 1 \\
14 & 1 & 1 & 1 & 0 & 39 & 3046 & 1 & 1 \\
15 & 1 & 1 & 1 & 1 & 0 & 0 & 0 & 1 \\
\hline
\end{tabular}
index.

Equation (17) shows the second contribution to the SAIDI

$$
S A I D I_{Z 89-S T A G E 2}=5.259 \mathrm{~min}
$$

Equation (18) shows the switching sequence $p_{2 s}$, automatically defined by the solution path.

$$
p_{2 s}=\{[89 \text { 90], [45 90], [53 96], [46 47] }\}
$$

The first couple of operations are to open switch [89 90] and close tie breaker [45 90], that allow the zone 90 to be supplied. Switch operations [53 96] and [46 47] complete the restoration process: load transfer from feeder $b$ to feeder $c$ move the grid topology in the final restoration scheme

The last contribution is related to the time required for the fault repair, i.e. the MTTR of the selected zone (240 minutes). The contribution, reported in equation (19), depends on the last state of the restoration process. In case of full restoration the third contribution is equal to zero.

$$
S A I D I_{Z 89-M T T R}=3.871 \mathrm{~min}
$$

Equation (20) shows the total contribution of the zone 89 fault to the global SAIDI index, considering the best switching sequence.

$$
S A I D I_{Z 89}=9.405 \mathrm{~min}
$$

The entire procedure can be performed setting ASIDI as target index. Results section shows the effect of the different target setting.

After the evaluation of the contributions for each zone fault, it is possible to compute the global indices using equation (11).

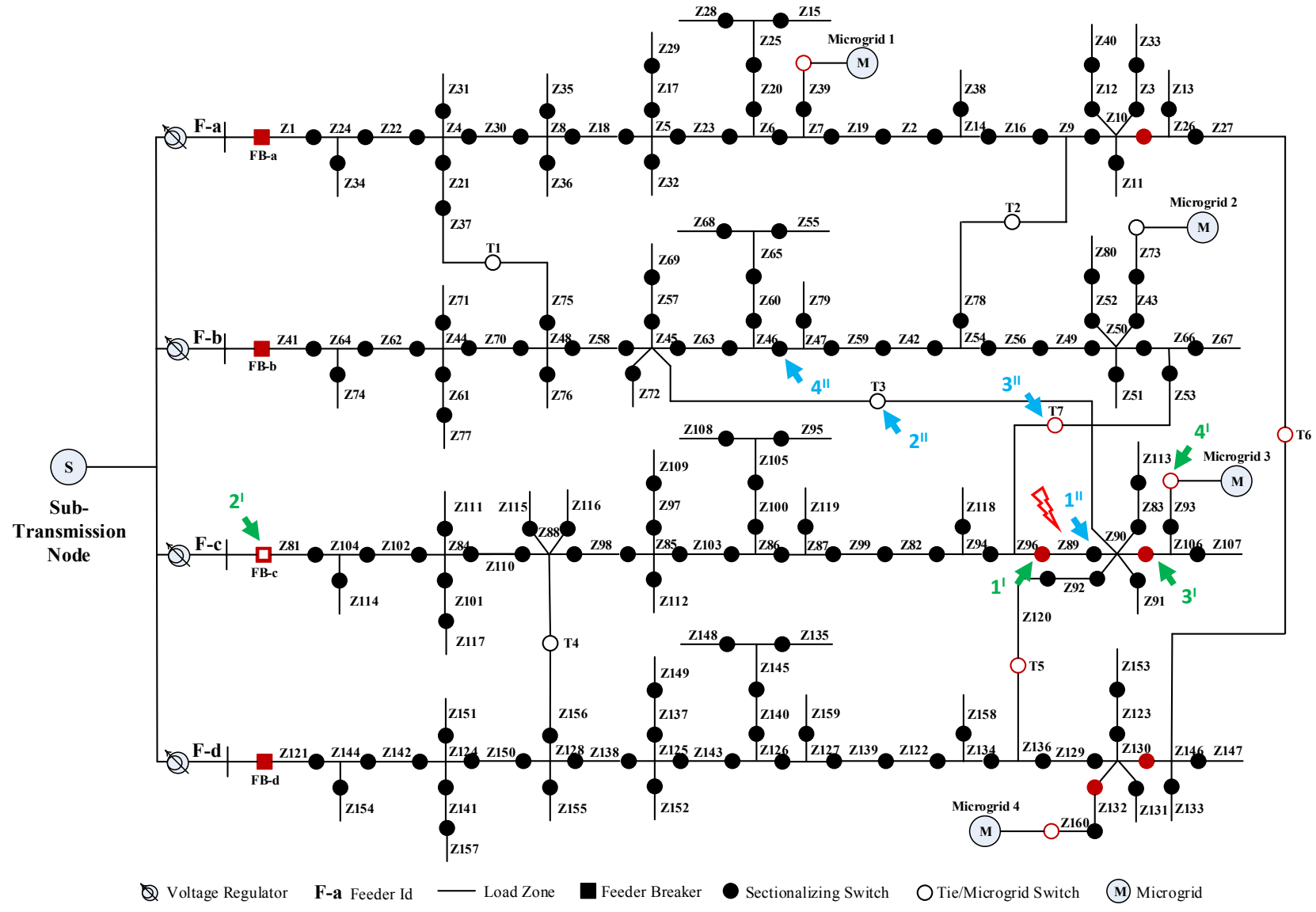

Figure 5 - The 4-feeder 1069-node test system 
After the best path determination, all the indices proposed in section II can be computed similarly to the target index, as sum of contribution definined by the restoration process steps.

\section{RESULTS}

The RA tool has been tested on the 4-feeder 1069-node test system, previously introduced. Table IV summarizes the main parameters adopted. Constant failure rates and MTTRs are considered for all the zones of the grid. The reliability evaluation is performed over one year of service, assuming constant time for the switching operation, 30 minute for manual switches and 20 seconds for RCs.

TABLE IV - STAGE 2

\begin{tabular}{ccccc}
\hline $\begin{array}{c}\text { FAILURE } \\
\text { RATE }\end{array}$ & MTTR & PERIOD & $\begin{array}{c}\text { MANUAL } \\
\text { SWITCH }\end{array}$ & $\begin{array}{c}\text { RC } \\
\text { SWITCH }\end{array}$ \\
\hline $0.021 /$ year & $240 \mathrm{~min}$ & 1 year & $30 \mathrm{~min}$ & $20 \mathrm{sec}$ \\
\hline
\end{tabular}

Table $\mathrm{V}$ reports the RA results obtained adopting the SAIDI index as a target. The availability of RC switches shows the improvement of system reliability. SAIDI and ASIDI indices show a reduction of $49.9 \%$ and $44.0 \%$, respectively. SAIFI and ASIFI indices are reduced as well, due to the exclusion of the contribution associated to customers that experience less than 5 minutes of interruption.

\begin{tabular}{lcccccc} 
& \multicolumn{6}{c}{ TABLE V-SCENARIO 1: SAIDI TARGET } \\
\cline { 2 - 7 } & SAIFI & SAIDI & ASIFI & ASIDI & CAIDI & ASAI \\
\hline no RCs & 0.800 & 57.570 & 0.800 & 60.815 & 71.962 & 0.9934 \\
RCs & 0.625 & 28.712 & 0.535 & 26.747 & 45.951 & 0.9967 \\
\hline
\end{tabular}

Table VI reports the RA results obtained adopting the ASIDI index as a target. RCs availability shows a reduction of $49.2 \%$ and $44.1 \%$ of SAIDI and ASIDI indices, respectively. Results are comparable with the SAIDI target scenario, due to the homogeneity between the number of customers and total contractual power of each zone.

TABLE VI - SCENARIO 2: ASIDI TARGET

\begin{tabular}{lcccccc} 
& SAIFI & SAIDI & ASIFI & ASIDI & CAIDI & ASAI \\
\hline no RCs & 0.800 & 58.449 & 0.800 & 60.310 & 73.061 & 0.9933 \\
RCs & 0.625 & 28.751 & 0.535 & 26.573 & 46.014 & 0.9967 \\
\hline
\end{tabular}

It is important to note that SAIFI and ASIFI indices of the two scenarios are the same. These two indices are related to the number and the power of interrupted customers (over 5 minutes of interruption). As a consequence, the values are related to the fault location. The effect of the restoration process is significant only for the first 5 minutes, i.e. the first stage. Further benefits can be obtained considering the increase of RC switches.

ASAI index appears to be closer to 1 in both scenarios. It represents the fraction of time that a customer has received power during the defined reporting period.

\section{CONCLUSIONS}

In this paper, a general methodology is proposed to evaluate reliability of electric distribution systems, moving from an historical data assessment to a computational approach. Service restoration is considered taking into account Microgrids availability.

The goal of the proposed methodology is to determine the optimal switching sequences for the restoration process, according to the target index. Six IEEE standard reliability indices are calculated, incorporating contribution of all possible fault occurrences.

Case study results show that the proposed method can be used to evaluate the benefits of Distribution Automation and smart grid applications.

\section{REFERENCES}

[1] K. Moslehi and R. Kumar, "A reliability perspective of the smart grid," IEEE Trans. Smart Grid, vol. 1, no. 1, pp. 57-64, Jun. 2010.

[2] C. L. Smallwood and J. Wennermark, "Benefits of distribution automation," IEEE Ind. Appl. Mag., vol. 16, no. 1, pp. 65-73, Jan.-Feb. 2010.

[3] H. Farhangi, "The path of the smart grid," IEEE Power Energy Mag., vol. 8, no. 1, pp. 18-28, Jan.-Feb. 2010.

[4] P. Wang and L. Goel, "Power distribution system reliability evaluation using both analytical reliability network equivalent technique and timesequential simulation approach," in Simulation Methods for Reliability and Availability of Complex Systems, London, UK: Springer, 2010, ch. 7, pp.145-172.

[5] R. Billinton and R. N. Allan, Reliability Evaluation of Power Systems, 2nd ed. New York, NY, USA: Plenum Press, 1996.

[6] K. Durga Rao, V. Gopika, V. V. S. Sanyasi, H. S. Kushwaha, A. K. Verma, and A. Srividya, "Dynamic fault tree analysis using Monte Carlo simulation in probabilistic safety assessment," Reliability Engineering \& System Safety, vol. 94, no. 4, pp. 872-883, Apr. 2009.

[7] DNV GL, Synergi Electric Software [online]. Available: https://www.dnvgl.com/services/power-distribution-analysis-andoptimization-synergi-electric-5005

[8] A. Coelho, A. B. Rodrigues, R. B. Prada, and M. G. Silva, "Reliability evaluation of distribution networks considering optimization models in the restoration process," in Proc. $10^{\text {th }}$ Int. Conf. Probabilistic Method Appl. Power Syst., Rincon, 2008, pp. 1-7.

[9] W. Li, P. Wang, Z. Li, and Y. Liu, "Reliability evaluation of complex radial distribution systems considering restoration sequence and network constraints," IEEE. Trans. Power Del., vol. 19, no. 2, pp. 753-758, Apr. 2014.

[10] P. Wang and W. Li, "Reliability evaluation of distribution systems considering optimal restoration sequence and variable restoration time," IET Gener. Transm. Distrib., vol. 1, no. 4, pp. 688-695, Jul. 2007.

[11] Y. Xu, C.-C. Liu, and H. Gao, "Reliability analysis of distribution systems considering service restoration," in proc. IEEE Innovative Smart Grid Technologies Conference (ISGT), Washington, DC, USA, Feb. 2015, pp. 1-5.

[12] Y. Xu, C.-C. Liu, K. P. Schneider, and D. T. Ton, "Placement of remotecontrolled switches to enhance distribution system restoration capability," IEEE Transaction on. Power System, DOI: 10.1109/TPWRS.2015.2419616.

[13] IEEE Power \& Energy Society, "IEEE Guide for Electric Power Distribution Reliability Indices", Std 1366-2012

[14] J. Li, X.-Y. Ma, C.-C. Liu, and K. P. Schneider, "Distribution system resotoration with microgrids using spanning tree search," IEEE Trans. Power Syst., vol. 29, no. 6, pp. 3021-3029, Nov. 2014.

[15] K. A. Ravindra, T. L. Magnanti, J. B. Orlin, "Network Flows - Theory, algorithms and applications", Prentice Hall, 1993

[16] E.W. Dijkstra, "A note on two problems in connexion with graphs", pp. 269-271, Numerische Mathematik 1,1959

[17] U.S. Department of Energy at Pacific Northwest National Laboratory, GridLAB-D, Power Distribution Simulation Software [Online]. Available: http://www.gridlabd.org/ 